decreases dynamic hyperinflation, and prolongs exercise tolerance. ${ }^{3}$

\title{
Combination therapy for exercise intolerance in COPD
}

\section{R Casaburi}

\section{New combined approaches to reduce dynamic hyperinflation improve exercise intolerance in patients with COPD}

C hronic obstructive pulmonary disease (COPD) is a miserable disits most troubling symptoms. Moreover it has recently been discovered that poor exercise tolerance is highly predictive of poor survival. Those who care for these patients are therefore highly motivated to seek ways to ameliorate exercise intolerance. In recent years progress has come from what, at first glance, would seem to be an unlikely quarter. Although research expenditures for this disease are heavily weighted towards cell and molecular biology pursuits, physiological research (considered "old fashioned" in some quarters) has provided solid insights into rational treatment for exercise intolerance.

A key insight is that dynamic hyperinflation is a major mechanism limiting exercise tolerance in patients with COPD. This occurs when the increased metabolic requirements of exercise demand higher levels of pulmonary ventilation. This, in turn, requires that both tidal volume and respiratory rate increase. The patient with COPD is asked to exhale a larger volume in a shorter time, which becomes an impossible task given flow limitation imposed by the increased expiratory airflow resistance. At some point the exhalation cannot be completed in the allotted expiratory time, full exhalation cannot be accomplished, and end expiratory volume increases. Eventually, dynamic hyperinflation leads to end inspiratory lung volumes that approach a limiting value (total lung capacity). As the inspiratory reserve volume shrinks, additional inspiratory volume cannot be achieved without greatly increased elastic work of breathing, since the lung is increasingly on a shallow portion of its pressure-volume curve. Severe dyspnoea results and exercise terminates when this sensation becomes unbearable.

\section{THERAPEUTIC APPROACHES}

Pulmonary physiologists have been working to develop strategies to lessen dynamic hyperinflation at a given amount of exercise. Logically, approaches that either allow faster exhalation (reduced expiratory airflow resistance) or prolong the time allowed for exhalation (decreased respiratory rate) will allow fuller exhalation, thereby reducing dynamic hyperinflation and improving exercise tolerance. To date, four distinct approaches have been defined that either lower expiratory airflow resistance or slow respiratory rate:

- Oxygen supplementation.

- Treatment with bronchodilators.

- Heliox (79\% helium, 21\% oxygen).

- High intensity rehabilitative exercise training.

\section{Oxygen supplementation}

Oxygen supplementation reduces carotid body drive and slows respiration at a given level of exercise. Dynamic hyperinflation is reduced and exercise tolerance is substantially prolonged. Increasing inspired oxygen fraction up to roughly $50 \%$ confers progressively greater benefits. This occurs even in patients with COPD whose level of exercise desaturation does not meet the usual criteria for oxygen supplementation.

\section{Treatment with bronchodilators}

COPD is now recognised as distinctly not a disease of irreversible airflow obstruction. In a substantial majority of patients with COPD treatment with bronchodilators yields clinically useful improvements in expiratory airflow, allows fuller exhalation in a given period of time, reduces dynamic hyperinflation, and prolongs exercise tolerance. Reduced dynamic hyperinflation accompanied by exercise tolerance improvements has been best demonstrated with long acting anticholinergic agents. $^{2}$

\section{Heliox}

Heliox (79\% helium, $21 \%$ oxygen) has a lower density than air and decreases airflow resistance. Breathing heliox lowers expiratory airflow resistance,
Rehabilitative exercise training

High intensity rehabilitative exercise training has been widely acknowledged to substantially increase exercise tolerance in patients with COPD. ${ }^{4}$ An important mechanism of the benefits of exercise training has recently been defined. ${ }^{5}$ As the exercising muscles become more fit, they release less lactic acid at a given level of exercise. Since lactic acid stimulates respiration, after rehabilitative training exercise ventilation is lower, respiration is slowed, and dynamic hyperinflation is reduced. ${ }^{5}$

\section{COMBINATION THERAPY}

If any one of these interventions improves exercise tolerance, it seems logical that a combination of any two ("combination therapy") would yield even better results. There seems to be a minor competition among physiologically orientated laboratories to see which can demonstrate the effectiveness of these four treatments taken pairwise.

Our laboratory has shown that providing supplemental oxygen $(3 \mathrm{l} / \mathrm{min}$ by nasal cannula) during a 7 week high intensity rehabilitation programme increased the tolerated work rates of patients during training and enhanced the improvements in exercise tolerance in comparison with COPD patients who trained while breathing room air. ${ }^{6}$ Patients who before training tolerated an average of 6 minutes of exercise at a given work rate breathing air tolerated an average of 26 minutes at the same work rate while breathing 30\% oxygen after an oxygen supplemented training programme. For several participants it was apparent that, after training, their physiological capacity for exercise had improved to such a degree that the assigned work rate was now below the critical power; ${ }^{7}$ they were able to tolerate the work rate essentially indefinitely.

Peter Calverley's laboratory investigated the combination of helium and oxygen breathing. In a crossover design, patients with COPD performed an endurance shuttle walk test while breathing air, $28 \%$ oxygen in nitrogen, $21 \%$ oxygen in helium, or $28 \%$ oxygen in helium. ${ }^{8}$ The latter mixture (high helium, high oxygen) yielded the highest shuttle walk distance. Whether $28 \%$ oxygen and $72 \%$ helium represents the optimal high oxygen/low density mixture for improving exercise tolerance remains to be determined.

A multicentre study showed that the benefits of a rehabilitative treadmill training programme were enhanced in a group of COPD patients receiving an effective long acting anticholinergic 
bronchodilator compared with a group receiving placebo.' By the end of the training programme, the duration of a constant work rate treadmill task was increased by $79 \%$ in patients receiving bronchodilator treatment compared with those receiving placebo.

Finally, in this issue of Thorax, Peters et al report that $50 \%$ oxygen breathing acts synergistically with bronchodilator therapy (ipratropium bromide) to reduce hyperinflation and improve exercise tolerance in patients with COPD. ${ }^{10}$ In a crossover study in 16 patients, the mean tolerance of a constant work rate increased by $57 \%$ and $31 \%$ when inhaling oxygen or receiving bronchodilator, respectively. The combination of oxygen and ipratropium increased exercise tolerance by an average of $87 \%$ over the control condition, demonstrating an approximately additive response.

The mathematically inclined among us will note that only four of six possible permutations of these four interventions have so far been reported. The successful combinations of heliox with exercise training and of bronchodilators with heliox are waiting to be achieved. Further, can studies of the simultaneous application of three interventions be far behind? Four studies of this kind could be planned, the requirement being the necessity to prove the superiority of the exercise tolerance improvements in response to three interventions over that of three groups in which combinations of two of the three interventions are applied. Will we see the day when a study appears in which simultaneous application of all four of these physiological manoeuvres are combined to ameliorate exercise intolerance and are proved to be superior to three groups in which three of the four interventions are given? One can only dream.

Thorax 2006;61:551-552.

doi: 10.1136/thx.2006.058511

Correspondence to: Dr R Casaburi,

Rehabilitation Clinical Trials Center, Los Angeles Biomedical Research Institute at Harbor-UCLA Medical Center, 1124 W Carson Street, Building J4, Torrance, CA 90502, USA; casaburi@ucla.edu

Funding: Dr Casaburi is the Grancell/Burns Chair in the Rehabilitative Sciences.

Competing interests: Dr Casaburi is a member of the speaker's bureau and is a consultant to Boehringer-Ingelheim Pharmaceuticals and Pfizer Pharmaceuticals.

\section{REFERENCES}

1 Somfay A, Porszasz J, Lee SM, et al. Doseresponse effect of oxygen on hyperinflation and exercise endurance in non-hypoxemic COPD patients. Eur Respir J 2001;18:77-84.

2 O'Donnell D, Flüge T, Gerken F, et al. Effects of tiotropium on lung hyperinflation, dyspnoea and exercise tolerance in COPD. Eur Respir $J$ 2004;23:832-40

3 Palange $P$, Valli $G$, Onorati $P$, et al. Effect of heliox on lung dynamic hyperinflation, dyspnea and exercise endurance capacity in COPD patients. J Appl Physiol 2004;97:1637-42

4 Troosters TR, Casaburi R, Gosselink R, et al. Pulmonary rehabilitation in chronic obstructive pulmonary disease. Am J Respir Crit Care Med 2005;172:19-38.

5 Porszasz J, Emtner M, Goto S, et al. High intensity training decreases exercise-induced hyperinflation in patients with COPD. Chest 2005; 128:2025-34.

6 Emtner M, Porszasz J, Burns $M$, et al. Benefits of supplemental oxygen in exercise training in nonhypoxemic chronic obstructive pulmonary disease patients. Am J Respir Crit Care Med 2003; 168:1034-42

7 Casaburi R. Factors determining constant work rate exercise tolerance in COPD and their role in dictating the minimal clinically important difference in response to interventions. $J$ COPD 2005;2:132-6.

8 Laude EA, Duffy NC, Baveystock C, et al. The effect of helium and oxygen on exercise performance in COPD: a randomised crossover trial. Am J Respir Crit Care Med 2006; 173:865-70

9 Casaburi R, Kukafka D, Cooper CB, et al. Improvement in exercise tolerance with the combination of tiotropium and pulmonary rehabilitation in patients with COPD. Chest rehabilitation in patien

10 Peters MM, Webb KA, O'Donnell DE. Combined physiological effects of bronchodilators and hyperoxia on exertional dyspnoea in normoxic COPD. Thorax 2006;61:559-67.

\section{COX-2: a link between airway inflammation and disordered chloride secretion in cystic fibrosis?}

\section{A Clayton, A J Knox}

\section{Possible role for COX-2 in the pathophysiology of CF}

L ung disease in cystic fibrosis (CF) continues to be the major cause of morbidity and mortality, with the mechanisms whereby the biochemical defect causes lung disease receiving considerable attention. CF is caused by mutations in a $230 \mathrm{kB}$ gene located on chromosome 7 which codes for the cystic fibrosis transmembrane conductance regulator (CFTR). ${ }^{1}$ CFTR functions in the main as a cAMP regulated chloride channel in epithelial and glandular structures. ${ }^{2}$ It has long been recognised that the abnormal chloride secretion by bronchial epithelial cells in CF predisposes to the development of bronchial damage and inflammation. The mechanism is under debate, but altered biochemical constituents of airway surface liquid, ${ }^{3-5}$ function of the mucociliary escalator, ${ }^{6}$ and the function of antibacterial defence molecules such as defensins ${ }^{6}$ are all thought to play a part. What is less clear is whether inflammation itself can feed back to further compromise the abnormalities in chloride transport leading to an amplificatory cycle of lung destruction. Studies of nasal potential difference in patients with CF have shown that there is a range in the severity of chloride flux reduction. ${ }^{78}$

Lipid mediators such as prostanoids have an important role in regulating inflammation in most tissues including the lung. Prostanoids are produced from membrane phospholipids by a three step reaction involving arachidonic acid release via phospholipase $\mathrm{A} 2$, conversion of arachidonic acid into $\mathrm{PGH}_{2}$ by cyclooxygenase (COX), followed by its conversion to terminal prostaglandins such as $\mathrm{PGE}_{2}$ by specific synthases and isomerases. COX exists in three isoforms: ${ }^{9}$ COX-1 is constitutively expressed; ${ }^{10} \mathrm{COX}$ 2 is inducible and therefore implicated in many inflammatory and malignant diseases, ${ }^{11}{ }^{12}$ and a further enzyme, COX-3, has recently been described whose function is less well defined. ${ }^{13}$ Previous studies have shown that the levels of prostanoids such as $\mathrm{PGE}_{2}, \mathrm{PGF}_{2}, \mathrm{PGF}_{1}$, and thromboxane $\mathrm{B}_{2}$ are raised in primary cultures of epithelial cells from the CF airway, ${ }^{14}$ suggesting that they may play a part in the pathogenesis of CF. Further evidence to suggest that prostanoids have an important pro-inflammatory role in CF is provided by clinical trials which have shown that the broad spectrum COX inhibitor ibuprofen can delay the progression of CF lung disease. ${ }^{15}$

Several questions are raised by these findings.

- What is the mechanism of increased prostanoid release and what is their role in regulating CF disease? 